\title{
A Cardiac Surgery Mini-Elective Increases Specialty Knowledge Acquisition Among Pre-Clinical Medical Students
}

\author{
Garrett Coyan $^{1}$, Maxwell Kilcoyne ${ }^{2}$, Edgar Aranda-Michel ${ }^{3}$, Derek Serna-Gallegos ${ }^{1}$, Angela \\ Kinnunen ${ }^{3}$, Arman Kilic ${ }^{2}$, and Ibrahim Sultan ${ }^{4}$ \\ ${ }^{1}$ University of Pittsburgh Medical Center \\ ${ }^{2}$ Medical University of South Carolina \\ ${ }^{3}$ University of Pittsburgh Medical Center Health System \\ ${ }^{4}$ University of Pittsburgh
}

June 28, 2021

\begin{abstract}
Background: The introduction of integrated thoracic surgery residency programs has led to increased recruitment efforts of medical students to pursue a career in cardiac surgery. With little representation of cardiac surgery in medical school curriculum, we assessed a cardiac surgery mini-elective's efficacy in improving perceived knowledge among medical students. Methods: Preclinical medical students were offered the opportunity to participate in a cardiac surgery mini-elective, which consisted of five 2-hour sessions. These sessions consisted of didactic and simulation components and covered topics including cardiopulmonary bypass (CPB) and extracorporeal membrane oxygenation (ECMO), aortic disease, aortic valve replacement (AVR), transplant and left ventricular assist devices (LVAD), and coronary artery bypass grafting (CABG). Students completed pre- and post-session survey's describing their perceived knowledge in these topics. Results: Overall, 22 students completed at least one session of the mini-elective. Fourteen $(73.7 \%)$ of the students were male. Fifteen $(68.2 \%)$ students completed at least three out of five sessions. The post-session survey responses showed significantly higher perceived knowledge compared to pre-session responses for all survey prompts of all five sessions. The CPB/ECMO and aortic disease sessions showed the greatest increase in post-session familiarity and perceived knowledge after the session $(\mathrm{p}<0.001)$ compared to the CABG, AVR, and transplant/LVAD sessions $(\mathrm{p}<0.05)$. Conclusions: Beyond developing interest in cardiac surgery, these data indicate that a well-planned didactic and surgical simulation program may build confidence in students' knowledge of various cardiac surgical topics. Further studies will need to address how this increase in perceived ability lasts over time and impacts career selection.
\end{abstract}

\section{Hosted file}

Mini_Elective_2020_v2.5.docx available at https://authorea.com/users/422329/articles/528060a-cardiac-surgery-mini-elective-increases-specialty-knowledge-acquisition-among-preclinical-medical-students 

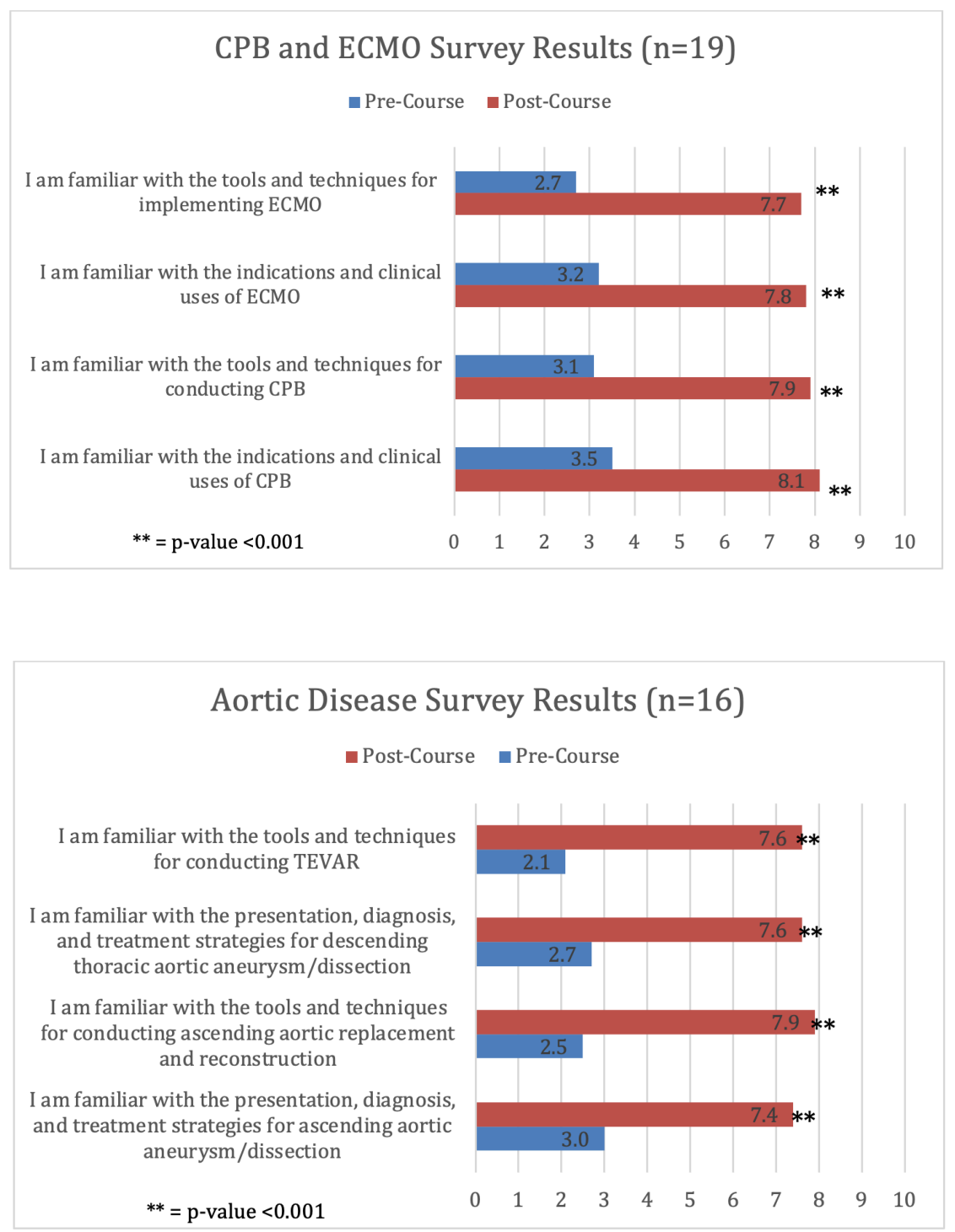

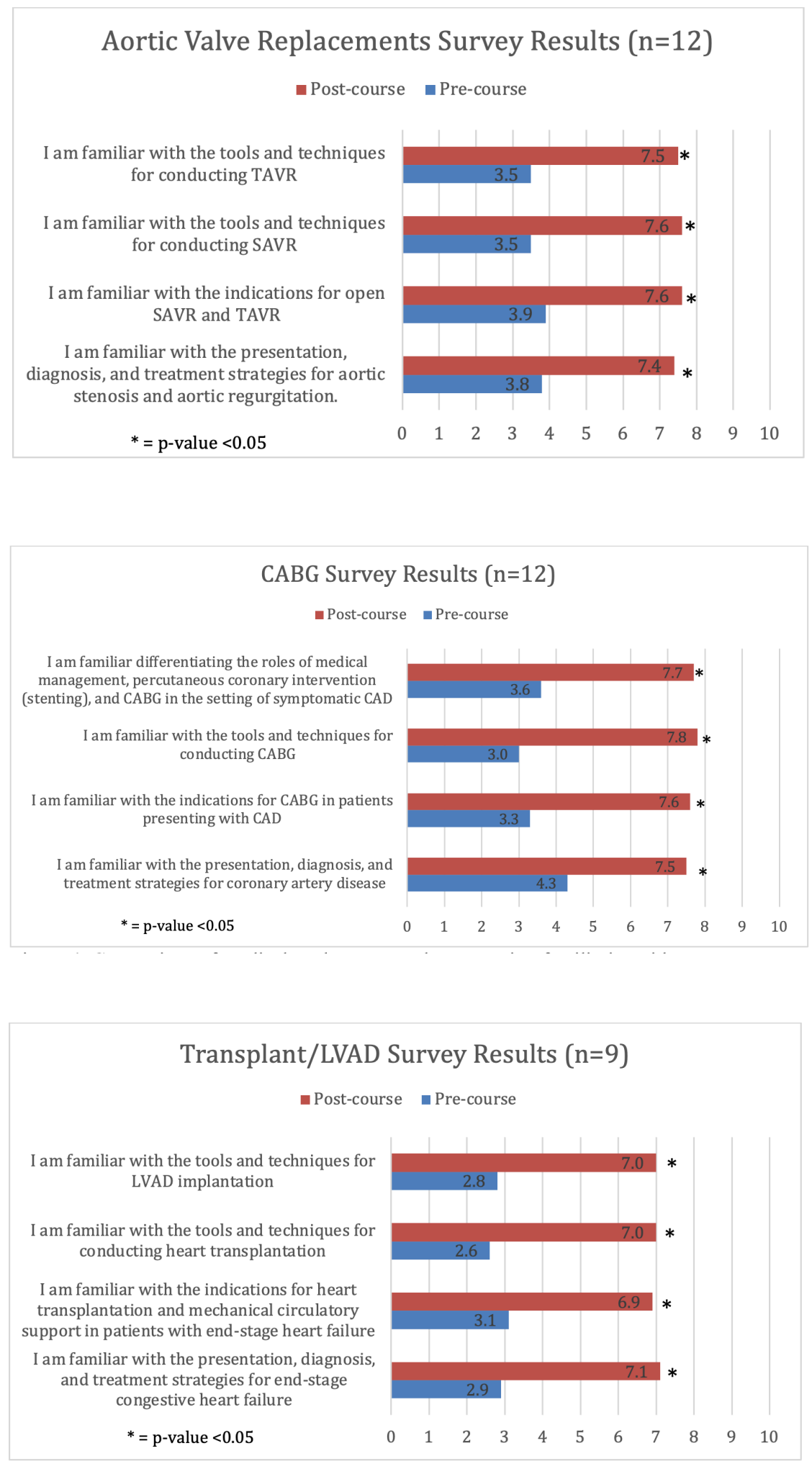